\title{
Ein Lehrstück helvetischer Polit- und Behördenkultur
}

\author{
K. Niedermann
}

In der NZZ, Nr. 53 vom 4. März 2004, Seite 53, erschien kürzlich folgender Kurzbericht mit wenig auffallender Schlagzeile:

\section{Bundesrat stützt Regierung bezüglich Privatspitaltarifen}

Urteil von der Praxis überholt

bto. Der Bundesrat hat in einem Tarifstreit zwischen der Zürcher Regierung auf der einen und den Privatspitälern und den Zürcher Belegärzten auf der anderen Seite zugunsten der Regierung entschieden. Demnach waren die Tarife richtig, welche der Regierungsrat den Privatspitälern im Juli 2000 für ambulante und teilstationäre Pflichtleistungen nach Krankenversicherungsgesetz vorgegeben hat. Verrechnet worden sind seither trotzdem teilweise höhere Tarife, welche das Eidgenössische Justiz- und Polizeidepartement den Privatspitälern in einem Zwischenentscheid zugestanden hatte.

$\mathrm{Zu}$ Unrecht, wie der Bundesrat jetzt laut einer Mitteilung der Gesundheitsdirektion entschieden hat. Allerdings hält er in seinem Entscheid fest, dass von einer Rückerstattung der Tarifdifferenzen ausnahmsweise abzusehen sei. Auf die heutige Praxis hat der Entscheid keinen Einfluss: Die umstrittene Regelung galt ohnehin nur bis zur Einführung des Abrechnungssystems Tarmed, was im Januar 2004 der Fall war. Auch in dieser Frage sind sich die Parteien nicht einig (NZZ 11.2.04). Eine Beschwerde der Privatspitäler ist beim Bundesrat pendent. Offen ist, ob auch deren Beantwortung drei Jahre beansprucht.

Dem NZZ-Redaktor stand für seine kurze Berichterstattung vermutlich nur die Pressemitteilung der Gesundheitsdirektion zur Verfügung. Als an der Beschwerde gegen den Zürcher Regierungsrat massgeblich mitbeteiligter Belegarzt drängt es mich, für die Leserschaft der Schweizerischen Ärztezeitung die Gründe der Beschwerdeeingabe von Zürcher Belegärzten und der Ärztegesellschaft des Kantons Zürich AGZ beim Bundesrat vertieft auszuleuchten.
Was sind die Gründe, die im Spätsommer 2000 zur Beschwerdeeingabe beim Bundesrat führten? (siehe auch [1]).

Dank Fortschritten in der operativen Medizin und Anästhesie ist es seit vielen Jahren möglich, zunehmend operative und diagnostische Eingriffe unter Spitalbedingungen (Infrastruktur, Fachpersonal) mit einer Hospitalisation unter 24 Stunden und ohne Übernachtung im Spital medizinisch zu verantworten. Voraussetzung dazu ist aber vorgängig eine gezielte Auswahl und Vorbereitung der Patienten (individuelle Eignung, soziales Umfeld, Behandlungs- und Betreuungsmöglichkeiten zu Hause) und eine entsprechende Ergebniskontrolle. So werden nach den neuesten Statistiken in den angelsächsischen und skandinavischen Ländern bis über 60 Prozent (USA) aller operativen Eingriffe in sogenannte Tageskliniken durchgeführt. Damit wird, wo medizinisch verantwortbar, eine «überlange Hospitalisationsdauer» vermieden. Dadurch können Kosten eingespart werden, ohne dass perioperativ an der Sicherheit und Qualität der medizinischen Leistung Abstriche gemacht werden.

Wie sieht diesbezüglich die Entwicklung in der Schweiz aus? Zuverlässige Zahlen fehlen, da im Schweizerischen Gesundheitswesen (UVG wie KVG) durch den Gesetzgeber (eidgenössisches Parlament) eine verbindliche Definition des kurzstationären $=$ tagesklinischen $=$ tageschirurgischen Bereichs bis heute nicht festgelegt worden ist (KVG-Revision II: Definition teilstationär?). Dazu kommt, dass die im KVG seit 1996 geforderte bundesrätliche Verordnung über die Kostenstellenrechnung und Leistungsstatistik in den Spitälern VKL erst seit Beginn 2004 in Kraft ist. Aufgrund dieser Verordnung gelten auch Hospitalisationen unter 24 Stunden mit einer Übernachtung im Spital als stationär (Bettenauslastung in den Spitälern). Die Leistungsstatistiken ambulant-stationär des Bundesamtes für Statistik sind daher bis 2004 in ihrer Aussagekraft mit entsprechender Vorsicht $\mathrm{zu}$ interpretieren.

Die geltenden Tarif- und Finanzierungsordnungen im föderalistischen schweizerischen Gesundheitswesen setzen praktisch keine Anreize für eine Tageschirurgie bei Ärzten, Spitä- 
lern, Versicherern und Versicherten. Gegenüber stationären weisen tageschirurgische Behandlungen einen ungenügenden Kostendeckungsgrad und mindestens bis 2004 eine geringere Honorierung der ärztlichen Tätigkeit bei identischer medizinischer Leistung auf. Für Versicherte und Versicherer ergeben sich die folgenden finanziellen Konsequenzen: Wird die Tageschirurgie dem ambulanten Bereich zugeordnet, erfolgt die Finanzierung nur über den Versicherer als alleinigen Kostenträger. Zusätzlich gehen der Selbstbehalt und die Franchise zu Lasten des Patienten. Wird die Tageschirurgie aber dem subventionierten stationären Bereich zugeordnet, muss sich der Versicherer nur bis maximal 50 Prozent an den stationären Kosten beteiligen. Der Rest wird vom Staat, d.h. vom Steuerzahler, getragen.

Für die Rechnungsstellung der tageschirurgisch erbrachten medizinischen Leistungen galten bis zur Einführung von TARMED im ambulanten und stationären Bereich unterschiedliche Tarifordnungen, die sich bei identischer Leistung betragsmässig stark unterschieden: im ambulanten Bereich nach Einzelleistungen mit einem Leistungskatalog, der sich ausschliesslich auf die Tätigkeit in nicht subventionierten Arztpraxen (Kanton Zürich: Krankenkassentarif) oder subventionierten Spitalambulatorien, Polikliniken und Notfallstationen (Kanton Zürich: Spitalleistungskatalog SLK mit einem um 20 Prozent reduzierten Taxpunktwert = Ambulatoriumstarif) ausrichtete; im subventionierten stationären Spitalbereich nach Tagesund Behandlungspauschalen (keine Vollkostendeckung).

$\mathrm{Zu}$ Zeiten des ehemaligen KUVG wurde nur zwischen den Begriffen «ambulant» und «stationär» unterschieden: Hospitalisationen, die kürzer als 24 Stunden waren, wurden als ambulant, solche, die länger als 24 Stunden dauerten, als stationär bezeichnet. Seit dem Wechsel zum KVG 1996 gibt es nun zusätzlich den Begriff «teilstationär», der im Gesetz aber nicht weiter definiert wird, so dass die Frage der Tarifierung und Finanzierung nicht geklärt ist. Damit ist es den Behörden (Kantone als Verordnungs- und Rekursinstanz, Subventionsverteiler und Spitalbetreiber) bzw. den Versicherern (Teil- oder Vollkostenträger) überlassen, den Bereich der kurzstationären Hospitalisation nach eigenem Gutdünken einzuordnen und damit die Tarif- und Finanzierungsordnung festzulegen.

1996 hat die Privatklinikgruppe Hirslanden in Zürich eine Tagesklinik eröffnet, die es ermöglichen sollte, auch grundversicherte Patien- ten des KVG im Rahmen der Tageschirurgie durch Belegärzte zu behandeln. Es stellte sich daher diesen freiberuflichen Ärzten die Frage, wie sie ihre in der Tagesklinik erbrachten Leistungen in Rechnung stellen können (gesplittete Rechnungsstellung): Der Krankenkassentarif für die Arztpraxis aus den siebziger Jahren war nicht leistungs- und sachgerecht (ungenügender Kata$\log$ für operative und anästhesiologische Leistungen) bzw. betriebswirtschaftlich ungenügend bemessen (nicht besoldete Belegarzttätigkeit im Privatspital). Der SLK-Tarif wurde von vielen Versicherern aber abgelehnt, da die Tageschirurgie für sie eine «ambulante Leistung» sei. Über die Ärztegesellschaft des Kantons Zürich wurde daher zur Problemlösung bei der Gesundheitsdirektion um die Einberufung eines «runden Tisches» nachgesucht. Die Versicherer lehnten aber ab, weshalb die Gesundheitsdirektion sich zunächst nicht weiter veranlasst fühlte, eine Einigung herbeizuführen.

Die meisten Belegärzte rechneten ihre Leistungen wie im UVG-Bereich nach SLK ab mit einem Taxpunktwert, der seit 1992 trotz Teuerung unverändert geblieben war. Die Höhe des Taxpunktwerts wurde von den Versicherern wiederum nicht akzeptiert: Der Wert hätte wie im öffentlich subventionierten Spitalbereich mit seinen besoldeten Spitalärzten um 20 Prozent tiefer $\mathrm{zu}$ liegen (Ambulatoriumstarif $=$ tariflich angeblich äquivalent zum Krankenkassentarif ohne Vorliegen von betriebswirtschaftlich erhobenen Vergleichsdaten), was die Belegärzte wiederum ablehnten. Der Leidtragende war einmal mehr der Patient, der von seiten der meisten Versicherer letztlich nur eine um 20 Prozent reduzierte Rückvergütung erhielt (Tarifschutz bei fehlenden Tarifverträgen?) abzüglich Selbstbehalt und Franchise.

Auf Veranlassung der Gesundheitsdirektion versuchte die Regierung des Kantons Zürich Anfang August 2000, die freiberufliche Ärzteschaft mit einem im Amtsblatt veröffentlichten und vorgängig nicht kommunizierten Regierungsratsbeschluss tariflich in die Pflicht zu nehmen, indem sie ohne Anhörung der Parteien (nicht subventionierte Leistungserbringer) den Ambulatoriumstarif des subventionierten Spitalbereichs auch für private nicht subventionierte Tageskliniken für verbindlich erklärte. Gegen diesen Beschluss haben Belegärztegruppen, die Ärztegesellschaft des Kantons Zürich und die Vereinigung der Zürcher Privatkliniken sogleich Beschwerde beim Bundesrat eingelegt in der Erwartung, dass von seiten des Bundesrats der Bereich Tageschirurgie nach den Kriterien des KVG endlich leistungs- und sachgerecht defi- 
niert und damit eine entsprechende Tarif- und Finanzierungsordnung für die Zukunft festgelegt wird.

Das Beschwerdeverfahren lag in den Händen des Bundesamts für Justiz im EJPD. Im Rahmen der Vernehmlassungen wurden neben den Beschwerdeführern zur Stellungnahme die Gesundheitsdirektion des Kantons Zürich, der Verband Zürcher Krankenversicherer VZKV (heute santésuisse Region ZH-SH), das Bundesamt für Sozialversicherungswesen BSV, die Preisüberwachung und die Paritätische Kommission «Spitalleistungskatalog» PKS eingeladen, nicht aber die FMH. Eigentlich wäre nach KVG dem Bundesrat für einen Entscheid nur maximal ein halbes Jahr zur Verfügung gestanden. Die Vernehmlassung wurde aber erst gegen Ende 2001 abgeschlossen. Der Schriftwechsel füllt bei jedem Beschwerdeführer mehr als zwei grosse Bundesordner.

In einem Zwischenentscheid des EJPD wurde den Beschwerdeführern die aufschiebende Wirkung zugestanden und damit erlaubt, für tageschirurgische Leistungen auf der Basis SLK mit nicht reduziertem Taxpunktwert weiterhin Rechnung zu stellen bis zur bundesrätlichen Entscheidung, spätestens aber bis zur Einführung von TARMED. Der Bundesrat hat erst Mitte Februar 2004 endlich entschieden (siehe oben zitierter Artikel in der NZZ), d.h. erst nach Einführung von TARMED.

Was hat die Einführung des TARMED in dieser nun «entschiedenen» Streitfrage für Auswirkungen? TARMED ist gemäss FMH (Urabstimmung) zunächst eine gesamtschweizerische Tarifstruktur für ambulante medizinische Leistungen sowohl für den Unfallversicherungswie für den Krankenversicherungsbereich des schweizerischen Gesundheitswesens, wobei für den Tarif der Taxpunktwert nach UVG gesamtschweizerisch, nach KVG aber kantonal festzulegen ist. Der Bundesrat hat sich in seinem Entscheid zur Begriffsdefinition «teilstationär» weiterhin nicht verbindlich geäussert. Die beiden eidgenössischen Kommissionen für soziale Sicherheit und Gesundheit haben sich im Rahmen der KVG-Revision II um eine Definition «teilstationär» mit unterschiedlichen Resultaten bemüht. Mit der Rückweisung der Revision im Nationalrat letzten Dezember sind für den Bereich Tageschirurgie mindestens für die nächste Zukunft keine weiteren Anreize geschaffen worden. In der Zwischenzeit hat die Gesund- heitsdirektion des Kantons Zürich von sich aus entschieden, dass teilstationär dem Begriff «ambulant» gleichzusetzen und daher mit TARMED abzurechnen ist. Damit legt sie zum Missmut der Versicherer und auch der Versicherten die Finanzierung der Kosten im Bereich «Tageschirurgie ohne staatliche Subventionen» wiederum fest und kann damit für den Staat zusätzlich Einsparungsmöglichkeiten auf Kosten des Privatspitalbereichs realisieren. So stehen wir wieder am Anfang des Beschwerdeverfahrens mit dem einzigen Unterschied, dass seit Januar 2004 nur noch eine gesamtschweizerisch einheitliche Tarifstruktur TARMED mit all ihren Unvollkommenheiten für die Honorierung der operativen belegärztlichen Tätigkeit existiert.

Über die rechtliche Qualität der Beweisführung für den bundesrätlichen Entscheid (gegen 70 Seiten) kann man als Nichtjurist kein Urteil abgeben. Wir haben den Entscheid zu akzeptieren, da im KVG keine Rekursmöglichkeit vorgesehen ist. Jedenfalls ist den Belegärzten für die Zeit seit Eröffnung der Tagesklinik Hirslanden im Sommer 1996 bis Ende 2004 keine Rückzahlungspflicht der Tarifdifferenz von 20 Prozent auferlegt worden. Ein Drittel des bezahlten Vorschusses der Verfahrenskosten wird ihnen rückerstattet. Es werden auch keine Parteienentschädigungen ausgesprochen. Am Schluss bleiben kostenmässig den Belegärztegruppen bezahlte Anwaltskosten von über Fr. 100 000.- für eine anspruchsvolle Rechtsvertretung vor dem Bundesrat. Ob diese Kosten mit der über drei Jahre zugestandenen Tarifdifferenz gedeckt sind, kann nicht beurteilt werden.

Für mich als standespolitisch aktiven Arzt geht damit neben dem vertieften Einblick in gesetzgeberische Mechanismen ein äusserst lehrreiches Kapitel bedächtiger helvetischer Politund Gesetzgebungskultur zu Ende. Mit dem KVG hat sich gezeigt, dass 1995 ein vom Gesetzgeber ungenügendes Gesetzeswerk dem Volk zur Annahme vorgelegt worden ist. Innert kurzer Zeit drängten sich die ersten Revisionen auf, die aber wegen ideologischer und parteipolitischer Auseinandersetzungen vom Parlament nur zum Teil durchgeführt worden sind. Die Beschwerdeeingabe machte zudem deutlich, dass in Sachen strikter Gewaltentrennung in der Schweiz nicht alles zum besten steht und dass die Frage nach einem Schweizerischen Verfassungsgericht in Zukunft bei den Politikern nicht mehr übergangen werden kann. 